\title{
Combining Evolutionary Inference and Metabolomics to Identify Plants With Medicinal Potential
}

\author{
Sundara M. U. P. Mawalagedera ${ }^{1}$, Damien L. Callahan ${ }^{2}$, Anne C. Gaskett ${ }^{3,4}$, \\ Nina Rønsted ${ }^{5}$ and Matthew R. E. Symonds ${ }^{1 *}$
}

${ }^{1}$ Faculty of Science, Engineering and the Built Environment, Centre for Integrative Ecology, School of Life and Environmental Sciences, Deakin University, Burwood, VIC, Australia, ${ }^{2}$ Faculty of Science, Engineering and the Built Environment, School of Life and Environmental Sciences, Deakin University, Burwood, VIC, Australia, ${ }^{3}$ School of Biological Sciences, University of Auckland, Auckland, New Zealand, ${ }^{4}$ Te Kura Mātauranga Koiora, Te Whare Wānanga o Tāmaki Makaurau, Auckland, New Zealand, ${ }^{5}$ Natural History Museum of Denmark, University of Copenhagen, Copenhagen, Denmark

OPEN ACCESS

Edited by:

Panagiotis Milonas,

Benaki Phytopathological

Institute, Greece

Reviewed by:

Joachim Ruther,

University of Regensburg, Germany

Leandros Alexios Skaltsounis,

National and Kapodistrian University

of Athens, Greece

*Correspondence:

Matthew R. E. Symonds

matthew.symonds@deakin.edu.au

Specialty section:

This article was submitted to

Chemical Ecology

a section of the journal

Frontiers in Ecology and Evolution

Received: 29 March 2019

Accepted: 25 June 2019

Published: 18 July 2019

Citation:

Mawalagedera SMUP, Callahan DL,

Gaskett AC, Rønsted N and

Symonds MRE (2019) Combining

Evolutionary Inference and

Metabolomics to Identify Plants With

Medicinal Potential.

Front. Ecol. Evol. 7:267.

doi: 10.3389/fevo.2019.00267
Plants have been a source of medicines in human cultures for millennia. The past decade has seen a decline in plant-derived medicines due to the time-consuming nature of screening for biological activity and a narrow focus on individual candidate plant taxa. A phylogenetically informed approach can be both more comprehensive in taxonomic scope and more systematic, because it allows identification of evolutionary lineages with higher incidence of medicinal activity. For these reasons, phylogenetics is being increasingly applied to the identification of novel botanic sources of medicinal compounds. These biologically active compounds are normally derived from plant secondary or specialized metabolites generally produced as induced responses and often playing a crucial role in plant defense against herbivores and pathogens. Since these compounds are typically bioactive they serendipitously offer potential therapeutic properties for humans, resulting in their use by traditional societies and ultimately drug lead development by natural product chemists and pharmacologists. The expression of these metabolites is likely the result of coevolutionary processes between plants and the other species with which they interact and effective metabolites are thus selected upon through evolution. Recent research on plant phylogeny coupled with metabolomics, which is the comprehensive analysis of metabolite profiles, has identified that related taxa produce similar secondary metabolites, although correlations are dependent also on environmental factors. Modern mass spectrometry and bioinformatic chemical networking tools can now assist high throughput screening to discover structurally related and potentially new bioactive compounds. The combination of these metabolomic approaches with phylogenetic comparative analysis of the expression of metabolites across plant taxa could therefore greatly increase our capacity to identify taxa for medicinal potential. This review examines the current status of identification of new plant sources of medicine and the current limitations of identifying plants as drug candidates. It investigates how ethnobotanic knowledge, phylogenetics and novel approaches in metabolomics can be partnered to help in characterizing taxa with medicinal potential.

Keywords: evolution, medicinal plants, metabolomics, phylogenetics, secondary metabolites 


\section{PLANTS AS A SOURCE OF MEDICINE}

Plants have been a perennial source of human therapeutics. The use of plants for medicine has been documented as early as 2600 BC (Gurib-Fakim, 2006) and plants still play a major role in treating human diseases. People in developing countries rely heavily on plant-derived traditional medicines (De Luca et al., 2012), accounting for close to $80 \%$ of world population (GuribFakim, 2006). In the United States, a considerable proportion of approved drugs are derived from plants $(9.1 \%$ according to the USFDA; United States Food and Drug Administration (Newman and Cragg, 2016). Furthermore, there are many drugs that are modified analogs of plant derived secondary metabolites that are in wide use (e.g., aspirin derived from the natural product salicin, from the willow tree Salix alba L., codeine and morphine from the opium poppy Papaver somniferum L.) (Dias et al., 2012). There are over 28,000 medicinal plants recorded in the scientific literature (Allkin, 2017) that are based on knowledge from traditional medicine.

Despite the prevalence of plants in traditional medicine, the majority of plant taxa have not received formal experimental appraisal for their medicinal properties. During the early twentyfirst century there has actually been a decline in the use of plants as a source of medicine in the pharmaceutical industry (Atanasov et al., 2015). Of the plant species recorded as having therapeutic properties, only $16 \%$ have been tested for biological activity (Willis, 2017). Furthermore, the pharmaceutical industry more commonly uses synthetic chemistry for drug design, even though success in finding new leads are limited (Li and Vederas, 2009) and there is a vast diversity of plants with biological activity that could be drug candidates. This decline is a result of the challenges faced in the conventional plant-based drug discovery process which make the overall process time consuming (Figure 1). The process starts with identifying the species of plant known to have medicinal function, sourcing the plant material, obtaining approval according to international legislations such as the convention on biological diversity and the Nagoya protocol on access to genetic resources, isolating the bioactive compound or compounds, identification of it, and ultimately synthesizing the candidate compound (Liu and Wang, 2008; Atanasov et al., 2015). For example, in the case of the drug artesunate derived from artemisinin originally discovered from Artemisia annua L., used to treat malaria, there was an 18 month lag between initial identification and production of the candidate drug. The time delay was due to the 6 month Artemisia cultivation period followed by the bioactive compound purification period (Wells et al., 2015). This was prior to the longer process of bioassaying, and before clinical testing. In addition to these practical issues, identifying the biologically active compounds from the plant source is challenging due to the enormous chemical diversity of plants and possibility of synergistic effects among compounds (Kingston, 2011). Furthermore, elucidating the mechanism of action of plant-based drugs also remains a slow process. These are only the delays after a plant source has been identified, and do not consider the time taken to identify that original botanic source in the first place. Overall this process of screening plant taxa for biological activity is costly, so the pharmaceutical industry tends to opt for other sources for medicines.

The study of therapeutic traditional use of plants is a multidisciplinary area, because both pharmacology of the plant and human behavior has affected whether and how the plant is used. As an example in traditional medicine there are many instances where complex herbal mixtures are used (Leonti and Casu, 2013). In such instances the metabolites in the herbal mixture may need to act in synergy to yield the pharmacological effects (Wagner, 2011). Further, some plant-based traditional medicines may also have different preparation methods. Based on the preparation methods the mode of action of the medicinal metabolites may differ (Heinrich et al., 2009). In addition to that some traditional communities may also select plants based on specific traits (De Medeiros et al., 2015). Therefore, consideration should be given to all of these related processes in ethnopharmacological studies. Despite these complications, ethnopharmacology still does provide a credible starting point in narrowing the target taxa for natural product chemistry research.

There are also significant challenges for managing how ethnopharmacological knowledge is partnered with scientific discovery methods and the pharmaceutical industry. Cultural appropriation and exploitation of traditional knowledge is a serious and ongoing issue given that intellectual property is often regulated by researchers and pharmaceutical companies (Mcgonigle, 2016). Thus a mechanism to acknowledge the indigenous knowledge of ethnobotany also needs to be adopted in drug discovery (Gupta et al., 2005). The International Platform on Biodiversity and Ecosystem Services (IPBES) established in 2012, acknowledges by developing and governing a set of principles that recognizes the contribution and knowledge of indigenous and local cultures in the use of biodiversity (Pert et al., 2015). Thus, the use of a proper legal framework and collaborations of indigenous communities, scientist and policymakers may also greatly help in sharing and utilizing the wealth of ethnopharmacological knowledge (Shane, 2004).

Largely in the pharmaceutical industry, drug discovery novelties are few and the need for bioactive compounds are persisting. Given the lengthy process of sampling, screening and biological testing, many plants are not investigated. Despite evidence that plants are a rich source of bioactive compounds. Hence the need for alternative approaches, to identify plants in a systematic manner for their medicinal potential is required. Novel approaches for identifying medicinal plant taxa more effectively need to be explored (Liu and Wang, 2008).

Recent advancements in the screening processes for natural products are stimulating renewed effort in plant-based drug discovery. Most notably, the 2015 Nobel Prize for medicine was awarded for identifying an antimalarial compound from Artemisia annua L. using the knowledge of traditional Chinese medicine ( $\mathrm{Tu}, 2011)$. Currently the two main drugs used to treat malaria are plant derived (artemisinin and quinine from Cinchona L. barks) highlighting that plant-derived pharmaceuticals are highly relevant for drug discovery. Furthermore, during the last decade, several new plant-derived drugs (apomorphine hydrochloride; water lily (Nymphaea L. sp), galanthamine hydrobromide; snowdrop (Galanthus nivalis L.), 


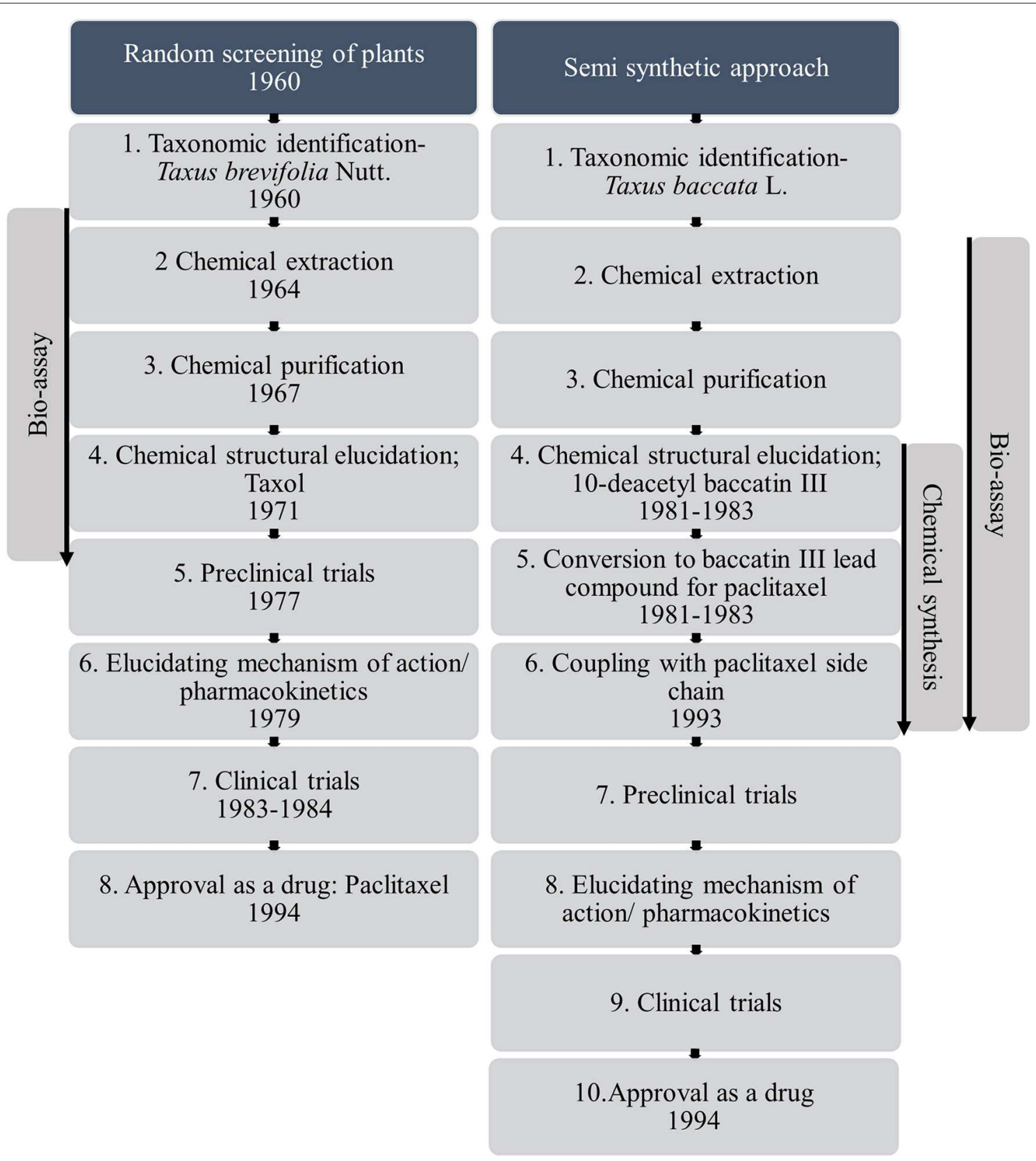

FIGURE 1 | Conventional pathway from random screening to novel drugs, using Taxus brevifolia Nutt. and alternative partially synthetic pathway from Taxus baccata L. as example species, a process which took over 30 years (based on information from Wani et al., 1971; Holton et al., 1994; Wall and Wani, 1995; Baloglu and Kingston, 1999; Liu and Wang, 2008; Weaver, 2014).

nitisinone; bottlebrush plant (Callistemon citrinus Curtis.), tiotropium bromide; devil's snare (Datura stramonium L.) and Cytisine; golden rain acacia (Cytisus laborinum L.) have been approved by the USFDA (Das, 2017; Auffret et al., 2018). According to World Bank projections, a growth rate of $5-15 \%$ annually in the plant-based medicinal market is anticipated (Liu and Wang, 2008). Thus, identifying biologically active potential medicinal plant sources is currently a commercially viable and beneficial research area, albeit one with significant cultural issues.
ROLE OF PLANT

\section{SECONDARY/SPECIALIZED} METABOLITES IN TREATING DISEASES

A medicinal plant is defined as a botanical source able to cure, prevent or relieve a disease, or a plant that is utilized as a drug or a precursor for a drug (Rates, 2001). Most of the active components of newly developed drugs are plant secondary metabolites (Balunas and Kinghorn, 2005). Plant secondary metabolites are 
compounds that are not directly responsible for plant growth and development, instead they are the products of specific sets of enzymatic reactions broadly known as metabolic reactions (Hartmann, 2007). Many plant secondary metabolites serve as defensive compounds produced to overcome the challenges plants face in the environment, including pathogens and herbivores but also abiotic stressors (Wink, 1988). In addition, some of these secondary metabolites also serve as allelopathic defensive compounds, synthesized in response to competition from surrounding plants (Reigosa and Pazos-Malvido, 2007). These chemical entities are specialized/secondary metabolites, which are typically expressed systemically, but occasionally the expression of these secondary metabolites is localized.

Plant secondary metabolites are structurally specialized by having highly active functional groups (i.e., aldehyde, sulfhydryl groups, epoxides, hydroxyl, and carbonyl groups) to act on cellular targets, such as enzymes, cell receptors and transporters. Therefore, when a medicinal plant product is ingested by humans, the secondary metabolites mediate a chain of reactions that may be beneficial in treating diseases (Acamovic and Brooker, 2005; Wink, 2015). As a result of their effect on the biological systems of hosts, secondary metabolites exhibit major properties as for example antiviral, antimicrobial, antifungal, antimalarial, analgesic, antiarrhythmic, antihypertensive, psychoactive, and tumor inhibition agents. However, out of c.100,000 known bioactive secondary metabolites, remarkably few have been scientifically screened for their activity (Wink, 2015).

The biggest challenge is identifying the target bioactive compound from the plant material, since every crude plant extract contains thousands of compounds, most of which are unknown (Atanasov et al., 2015). Thus, identification of biologically active compounds is done through comparing the chemical structures of compounds identified from the plant, to those already existing in compound libraries typically used in analytical processes (i.e., mass spectrometry (MS) and nuclear magnetic spectroscopy (NMR) (Dias et al., 2016). Examples of these include KNApSAcK (Afendi et al., 2012), the Universal Natural Product Database (UNDP) which is an open source library with bioactivity information, the Global Natural Product Database (GNPS) and Massbank (Johnson and Lange, 2015). However, these libraries do not include all the natural products that plants produce (Schwager et al., 2008). Furthermore, inevitably if searching for new candidate drugs, many plant compounds are likely to be new to science (Takeuchi et al., 2018), and hence not present in compound libraries. Therefore, studies providing broad identification of the secondary metabolites with biological activity from plants, can provide an enormous amount of information applicable to the discovery of new therapeutic plants (Harvey et al., 2015).

\section{IDENTIFYING THERAPEUTIC PLANTS ACROSS THE PLANT KINGDOM}

Known medicinal activity is not randomly distributed across the plant kingdom. There are specific distribution patterns where certain lineages are significantly more bioactive (Coley et al., 2003). This pattern is also reflected in the traditional use of plants by humans. Different cultures around the world tend to use related plant taxa for similar medicinal purposes (Saslis-Lagoudakis et al., 2011). This ethnobotanical convergence suggests that therapeutic potential may have phylogenetic constraints (Zhu et al., 2011 Kowiyou et al., 2015; Garnatje et al., 2017). Therefore, by investigating ethnopharmacological use of plants, phylogenetically related target species for further investigation could be identified.

Phylogenetic exploration of approved drugs of natural origin has identified 11 major plant clades that have delivered the significant part of these approved drugs (Zhu et al., 2011). Likewise, there is evidence of particular plant lineages expressing specific secondary metabolites with medicinal function (Rønsted et al., 2012). For example, in-depth studies of the synthesis of the secondary metabolites of terpene structure reveal that there are seven groups of gene families responsible for terpene synthesis. These genes are specific to plant lineages (land plants, vascular plants, gymnosperms) and the types of terpenes they synthesize varies, respectively between clades (Feng et al., 2011). Thus, a broader understanding of how the genes responsible for secondary metabolism have evolved across plant taxa will provide information on the type of secondary metabolites synthesized by a plant lineage.

Secondary metabolites can be either ubiquitous or expressed in specific plant lineages (Chezem and Clay, 2016). Since secondary metabolites confer survival benefits to plants, they are under natural selection (Wink, 2003). The genes responsible for plant secondary metabolism are versatile and have high plasticity to adapt to environmental pressures. In contrast, the genes responsible for primary metabolism are stringent and expression is generally relatively inflexible (Hartmann, 2007). Genes responsible for secondary metabolism therefore have the ability to be upregulated and downregulated across lineages in response to selection imposed by the other species with which they interact (e.g., herbivorous insects) or environmental stress factors. This presents extra challenges to drug discovery as environmental factors can also play a role in the amount of a particular bioactive compound present in a natural product extract.

Differential gene expression is responsible for the lineage specificity of secondary metabolites. For example, in Arabidopsis thaliana (L.) Heynh. and other closely related Brassicaceae plants, glucosinolate expression is controlled by the methylthioalkylmalate (MAM) synthases gene cluster (Benderoth et al., 2006). Studies of differential expression of MAM genes have shown that positive natural selection has shaped the synthesis of glucosinolates as a defense mechanism to insect herbivory (Benderoth et al., 2006; Figueiredo et al., 2008). The phylogenetic history of Arabidopsis and its close relatives reveals specific gene duplication points, where new MAM genes were positively selected for the combinations of glucosinolates that those genes encoded (Benderoth et al., 2006). Synthesis of many plant secondary metabolites is regulated by different gene families, and they could have similar underlying gene duplication and selection histories explaining their diversity. 
Differential gene expression in secondary metabolism is also driven by coevolution - the correlated evolution between two groups of ecologically interacting organisms, resulting in reciprocal evolution of both groups in response to each other (Ehrlich and Raven, 1964). Coevolution-mediated expression of secondary metabolites has been a major hypothesis in understanding the differential expression of secondary metabolites across plant lineages (Speed et al., 2015). One of the best studied models of coevolution concerns the chemical diversity of the family Apiaceae (which includes such wellknown plants as dill; Anethum graveolens L., coriander; Coriandrum sativum L., cumin; Cuminum cyminum L. and fennel; Foeniculum vulgare Mill.). The coevolution of the Apiaceae and Papilio butterflies is reflected in their shared patterns of phylogeny where chemical shifts in the phylogeny of the butterflies has governed host plant diversifications (Berenbaum, 2001; Berenbaum and Zangerl, 2008). Several other well-documented examples of reciprocal adaptations between host plants and their specialist herbivores include Barbarea vulgaris (Aiton) (rocketcress; Brassicaceae) and Phyllotreta nemorum L. (turnip flea beetle), Brassicales plants and Pierinae butterflies, and Pastinaca sativa L. (Parsnips; Apiaceae) and Depressaria pastinacella (Duponchel) (Parsnip webworm) (Zangerl et al., 2003; Toju and Sota, 2006; Agrawal et al., 2012b; Edger et al., 2015).

Plant secondary metabolite expression can also be driven by generalized, rather than specialized, herbivores. Recent research on Amazonian trees (from the tribe Protieae; Burseraceae) showed that secondary metabolites able to act as herbivore repellents were found frequently in high abundances in Protieae tribe plants, even in the absence of specialized herbivores. This suggests that regardless of the type of herbivore, the mere existence of any natural enemy can affect how secondary metabolites are expressed in plants (Salazar et al., 2018). Conversely, Oenothera biennis L. (common evening-primrose) populations grown in controlled insect pest-free environments, had reduced defenses against insects pests over time, i.e., they reduced their production of toxic secondary metabolite in response to decreased herbivory (Agrawal et al., 2012a).

Some of the chemical drivers behind the expression of these secondary metabolites are herbivore-associated elicitors (metabolites released into the plant by the herbivores). Many of these elicitors are produced by insects, and range from enzymes and modified lipids to sulfur containing fatty acids (Bonaventure et al., 2011). These elicitors have the ability to stimulate biosynthetic pathways within a plant (Waterman et al., 2019). Further, the action of these elicitors tends to be associated with certain plant-insect associations. For example the lepidopteran Manduca sexta L. (tobacco hornworm) releases fatty acid-amino acid conjugates into the plant that stimulate the biosynthesis of jasmonic acid in Nicotiana attenuata Torr. ex S.Watson (wild tobacco) (Wu et al., 2007; Meldau et al., 2011). Jasmonic acid afterwards mediates the synthesis of secondary metabolites as defense compounds in $N$. attenuata (Kallenbach et al., 2010). Thus, expression of secondary metabolites is governed both by evolution (i.e., one-way adaptation in response to generalized herbivory) and coevolution (i.e., specific reciprocal adaptation between the interacting host and a herbivore species) (Caseys et al., 2015).

Overall, the genetic mediation of plant secondary metabolite expression does not act in isolation. There are abiotic factors such as temperature, soil $\mathrm{pH}$, and frost exposure that may have effects on overall plant secondary metabolism. In Zea mays L. (corn) changes in abiotic factors as soil humidity, temperature and light had significant effects on the volatile secondary metabolite production (Gouinguen and Turlings, 2002). These environmental stresses are also correlated with the abundance of natural enemies or herbivores and plant pathogens which themselves affect the expression of secondary metabolites (Müller and Orians, 2018). Therefore, studying secondary metabolite expression, in the light of coevolution would provide a more holistic and biologically relevant method to understand differential gene expression, and thus the distribution and occurrence of plant secondary metabolites. This has theoretical implications for the tracking the expression of traits according to phylogeny, as well as for medicinal and drug discovery.

\section{USE OF PHYLOGENY AND METABOLOMICS TO IDENTIFY BIOLOGICAL ACTIVITY IN PLANTS}

Understanding how secondary metabolite gene expression varies across clades is a potential key to identifying biologically active plants. For example, the kinds of enzyme-encoding gene clusters used by algae and mosses are distinctively different from those found in grasses and eudicots (Chae et al., 2014). Furthermore, analysis of the metabolomic reactions within these plant groups has shown that closely-related taxa express the same set of reactions, thus suggesting phylogenetic constraints on secondary metabolite expression. If true, in depth analysis of plant secondary metabolite evolution would help us to understand which taxa/clades are biologically active, and hence are good candidates as medicinal species. In other words, the phylogenetic patterns of secondary metabolite expression could potentially act as a marker for biological activity, and therefore potential medicinal use.

There have been numerous studies in the past decade describing phylogenetic patterns of secondary metabolite expression. Analysis of volatile terpenes from angiosperm taxa has shown phylogenetically conserved expression, with related species producing the same terpenoid across the phylogeny. In particular, species belonging to the Magnoliid clade such as orders Laurales, Magnoliales had very high terpene diversity (Courtois et al., 2016). This suggests that the origin of the Magnoliids 122-125 million years ago is a key evolutionary point at which plant volatile terpene synthesis increased significantly. The Magnoliids are also one of the eleven clades predicted to have significantly higher pharmacological activity (Zhu et al., 2011). Indeed, over a quarter (18 families) of the 66 drug productive families identified, are from the Magnoliid clade (Zhu et al., 2011). Other lineages with high degrees of bioactivity are mosses from the order Hypnales, conifers (Pinales, was Coniferales), plus angiosperms from the monocots (Commelinids, and orders 


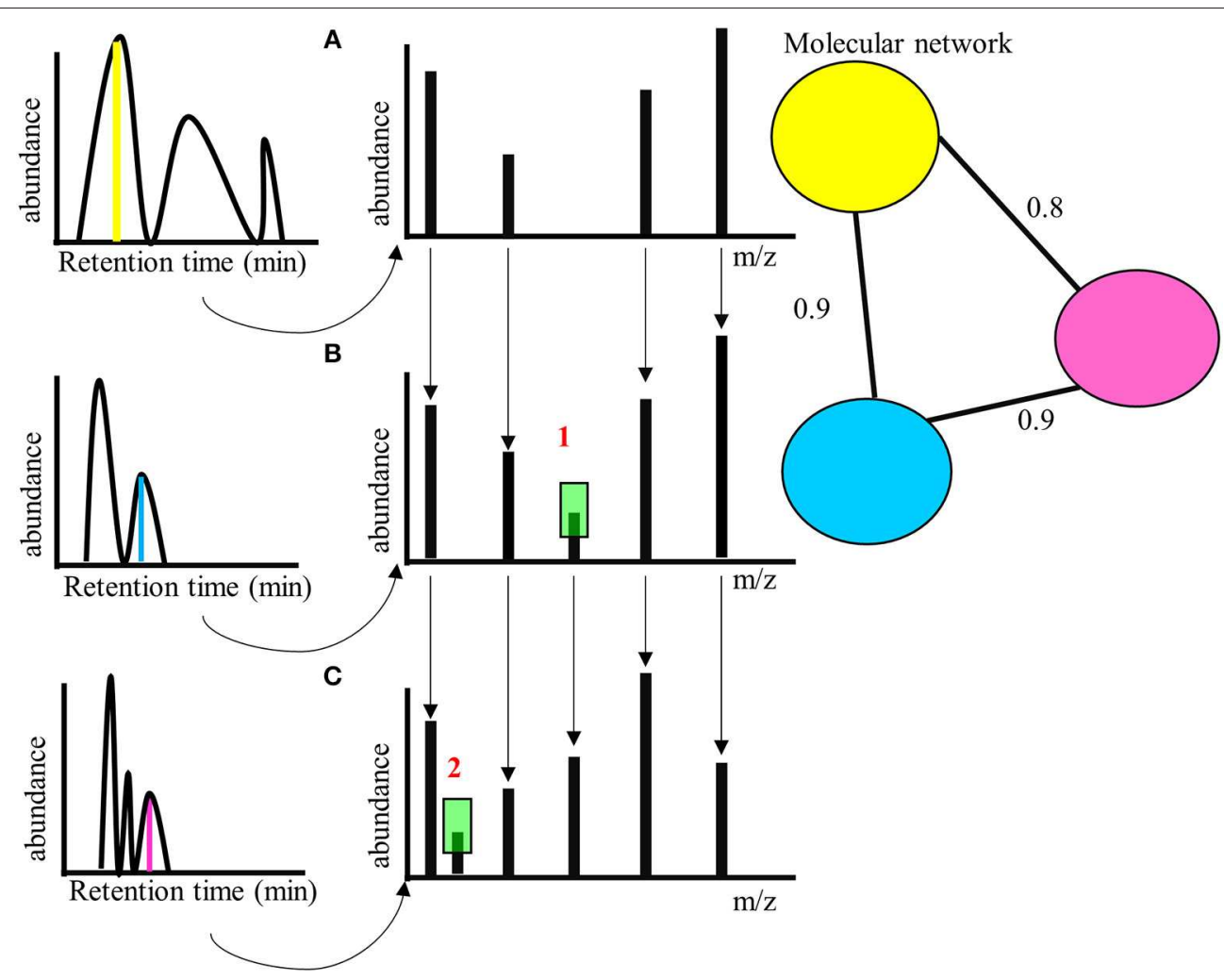

FIGURE 2 | (A) Hypothetical molecular network for three compounds eluting at different retention times, with different parent masses (left, indicated from the MS parental ion spectra in yellow, blue, and magenta) - but with similar MS/MS fragment ion spectra, right (A), (B), and (C). The similarity score is calculated for the MS/MS fragmentation patterns based on number of peaks that are similar across (A-C). 1 is a single peak difference between (A) and (B) (highlighted in green) - the similarity score of yellow and blue MS/MS fragment spectra is 0.9 .2 a single peak difference between (B) and (C) (highlighted in green) -the similarity score of blue and magenta MS/MS fragment spectra is also 0.9. 1 and 2 combined are two peaks difference between (A) and (C) - the similarity score of yellow and magenta MS/MS fragment spectra is therefore somewhat less $=0.8$. The molecular network is generated by calculating the similarity score between yellow, blue, and magenta compounds (parent ions- the nodes in the network) based on the MS/MS fragmentation (C), (B), and (A). The connecting lines (edges) gives the similarity score.

Threshold values of similarity can be set to determine presence within a network.

Asparagales and Liliales), the basal or early diverging eudicots (Ranunculales) and more derived eudicots (Fabids and Malvids from the Rosids, and Lamiids and Campanuliids from the Asterids) (Zhu et al., 2011); APG IV 2016). Phylogenetic patterns in bioactivity (through secondary metabolite expression) have also been examined at the genus level for the angiosperms Euphorbia (Euphorbiacae; Rosid) and Narcissus (Amaryllidaceae; Asparagales) with both genera yielding significant phylogenetic signals in biologically active secondary metabolite expression (Rønsted et al., 2008; Ernst et al., 2016).

The strong patterns of phylogenetically conserved expression of secondary metabolites could underlie the observed ethnobotanical convergence, in which similar plant taxa are used in similar ways in different parts of the world. Research on cross cultural patterns of medicinal plant usage reports the use of taxa from the same clades, across continents to treat similar diseases, signifying that there is phylogenetically non-random selection of therapeutic plants by indigenous cultures (SaslisLagoudakis et al., 2011, 2012). As an example, there are accounts for using Piper umbellatum L. (cow foot leaf; Piperaceae,
Magnoliid) to treat kidney and digestive diseases across North America, Africa and South East Asia (Roersch, 2010). Further cross-cultural patterns have been revealed in a larger study across a number of genera e.g., Aloe Aloe vera (L.) Burm. f. (Asphodelaceae; Asparagales), the breadfruit Artocarpus altilis (Parkinson) Fosberg (Moraceae; Rosid), Papaw Carica papaya L. (Caricaceae; Brassicales) and love vine Cassytha filiformis L. (Lauraceae, Magnoliid) used for medicinal preparations across the Caribbean Islands (Halberstein, 2005). Thus, information from traditional medicine, along with biological active metabolite expression, coupled with phylogeny could be the strategy for next generation drug productive taxa identification.

Recent research has used phylogenetic approaches to predict medicinal potential of plants. For example, phylogenetic mapping was performed for plant species with compounds used for treating cardiovascular disease, based on ethnobotanic and pharmacological mode of action of the drug. Seven angiosperm plant families were identified as having similar pharmacology: Zingiberaceae (Commelinid monocot), Brassicaceae, Fabaceae, Malvaceae, Rosaceae (Rosids), and Apiaceae and Lamiaceae 


\begin{tabular}{|c|c|c|}
\hline \multirow[t]{8}{*}{ A } & Taxa & Secondary metabolite \\
\hline & $\mathrm{A} \not \mathrm{P}$ & $\mathrm{X}, \mathrm{X} 1$ \\
\hline & $\mathrm{B}$ & $\mathrm{X} 1$ \\
\hline & $\overline{\mathrm{C}}$ & $\mathrm{X} 2$ \\
\hline & $\mathrm{D}$ & 8 \\
\hline & $\mathrm{P}$ & Y, Y1 \\
\hline & $Q \mathrm{Q}$ & $\mathrm{Y}$ \\
\hline & $\mathrm{R}$ & QP \\
\hline \multicolumn{3}{|c|}{ Q - known traditional medicinal taxa } \\
\hline \multicolumn{3}{|c|}{ Q - taxa with no identified secondary metabolite profiles } \\
\hline \multicolumn{3}{|c|}{$\mathrm{X}$ - known bioactive secondary metabolite } \\
\hline \multicolumn{3}{|c|}{ Y - known bioactive secondary metabolite } \\
\hline \multicolumn{3}{|c|}{$\mathrm{X} 1, \mathrm{X} 2$ - metabolites structurally similar to $\mathrm{X}$} \\
\hline \multicolumn{3}{|c|}{ Y1 - metabolites structurally similar to $\mathrm{Y}$} \\
\hline
\end{tabular}

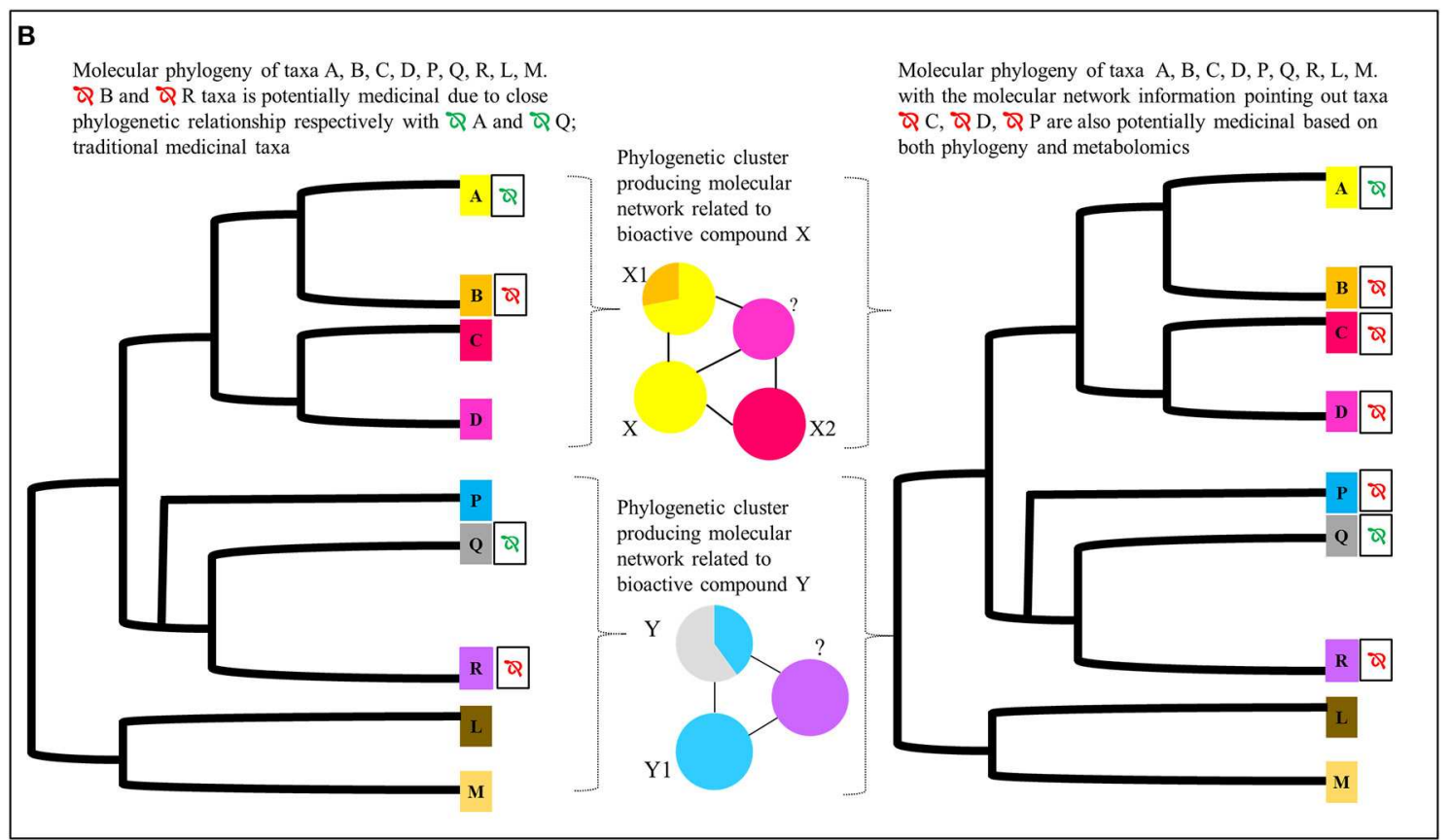

FIGURE 3 | Proposed integrated approach for identifying potentially medicinal plant taxa using metabolomics profiles of species A, B, C, and D (phylogenetically closely related species belonging to same operational taxonomic unit/family) and species $P, Q$, and $R$ (likewise but from a different operational taxonomic unit/family). (A) Hypothetical secondary metabolites identified using UHPLC-MSMS in respect to taxa A, B, C, D, P, and Q. Only taxa A and Q have previously known traditional medicinal uses. Taxa $A$ and $Q$ express the already identified bioactive metabolites $X$ and $Y$, respectively. Taxon $D$ is phylogenetically closely related to taxa $A, B$, and $C$. It does not have any known medicinal properties or identified bioactive metabolites. Taxon $\mathrm{R}$ is phylogenetically closely related to taxa $\mathrm{P}$ and $\mathrm{Q}$. It does not have known medicinal properties or identified bioactive metabolites. (B) Hypothetical GNPS based molecular network ${ }^{*}$ and the molecular phylogeny of the corresponding taxa. Q Potentially medicinal taxa. These taxa could be identified by looking at the molecular network cluster patters and the phylogenetic relationships of the target taxa $D$ and $R$ for which there are no previous records of metabolomic profiles. Given that their respective locations in relation to taxa $A, B, C, P$, and $Q$, statistical inferences could be used to predict the medicinal potential based on phylogeny and metabolomic features. ${ }^{*}$ n the molecular network the nodes are further divided in to pie charts to represent the taxa sharing a particular metabolite. The area corresponds to the parent ion intensities in each node produced by respective taxa. The color code of the nodes correspond to the color code on the metabolites table and the phylogeny for ease of understanding the figure.

(Asteriids). Those families have been suggested as potential target clades for identifying novel leads in treating cardiovascular diseases (Guzman and Molina, 2018). Despite these promising advances, research to date has not specifically quantified the capacity of phylogeny to predict medicinal potential in specific lineages. This can be done by measuring the phylogenetic signal of a trait (Münkemüller et al., 2012), in this case, biological activity, across a large clade of plants with a high frequency of reported traditional medicinal use.

Phylogenetic signal reflects the tendency of closely-related species to be phenotypically similar in contrast to species drawn at random from the same phylogeny (Blomberg et al., 2003). It can be measured via a number of metrics for data that are continuous (quantitative) or discrete (e.g., presence/absence 
data) (Fritz and Purvis, 2010; Münkemüller et al., 2012; Kamilar and Cooper, 2013). In a medicinal use context, Fritz and Purvis's (2010) D statistic has been used to compare the phylogenetic distribution of presence/absence of both therapeutic use and expression of particular compounds across plant species, and whether this conforms to either a random distribution (with no phylogenetic signal) or distribution that would be expected if the trait had evolved by Brownian motion (gradual divergent evolution) (Saslis-Lagoudakis et al., 2015). This has been used to identify a phylogenetic signal of medicinal potential in the family Amaryllidaceae in relation to alkaloid diversity, where five of seven alkaloid groups exhibited weak, but significant, phylogenetic signal (Rønsted et al., 2012). The $\mathrm{D}$ statistic was also used to map the phylogenetic signal in leaf succulence and medicinal use across the genus Aloe finding that a succulent leaf habit is associated with the potential of a species to be used as a medicine (Grace et al., 2015). Furthermore, the D statistic has also been used to predict presence of artemisinin and antimalarial properties in other Artemisia (other than Artemisia annua L. (Pellicer et al., 2018).

\section{USING PHYLOGENETIC TRAIT MAPPING AND MOLECULAR NETWORKING TO PREDICT MEDICINAL POTENTIAL}

Novel techniques of metabolomics such as molecular networking can be used to identify metabolites that are structurally similar to already known bioactive metabolites (Allard et al., 2016). In molecular networking the fragmentation pattern of a metabolite (Tandem mass spectrometry-MS/MS fragmentation) is compared with other compounds and similar metabolites are identified based on the structure (Figure 2). These are then put into network clusters, where a single cluster would therefore refer to a single metabolite group that likely share many similar chemical (and hence potentially bioactive) properties. Therefore, this technique allows one to identify groups of potentially bioactive metabolites, and novel compounds with such properties. Further, by treating such networks as phenotypic characters, one can map their expression onto phylogenies and identify clades with related bioactive properties in a way that does not rely specifically on the identification of single compounds.

Tandem mass spectrometry-MS/MS based molecular networking has been extremely informative in chemical similarity studies identifying related chemical entities across taxa/clades. Once the chemical entities are identified using Global Natural Product Social Molecular Networking (GNPS), it is possible to identify which taxa share the same molecular network cluster. Furthermore, MS/MS spectra can be related to

\section{REFERENCES}

Abbas-Mohammadi, M., Moridi Farimani, M., Salehi, P., Nejad Ebrahimi, S., Sonboli, A., Kelso, C., et al. (2018). Acetylcholinesterase-inhibitory activity of iranian plants: combined hplc/bioassay-guided fractionation, molecular biosynthetic pathways and their biological activity using online databases for natural products, thus providing information on the medicinal potential of a taxon. One limitation with this approach is that it requires some pre-existing knowledge of chemical compounds' biological activity to identify analogs with similar potential. It may not be so effective in identifying novel biologically active compounds that have completely undescribed chemical composition.

There have been numerous studies identifying novel bioactive candidate compounds using molecular networking in species such as in the Euphorbiacaee species Euphorbia dendroides L. (tree spurge) and Codiaeum peltatum (Labill.) P.S.Green (croton), and identifying chemicals of cannabinomimetic activity from the cyanobacteria genus Moorea (Kleigrewe et al., 2015; Nothias et al., 2018; Olivon et al., 2018). However, the focus of these studies was on single taxa without considering their evolutionary history. Multi-species studies do exist, as in the recent study of acetylcholinesterase inhibitors produced by a range of Iranian monocot and eudicot flowering plant taxa, where new compounds as well as taxa responsible for expressing the new compounds were identified (Abbas-Mohammadi et al., 2018), but again the study taxa here were restricted only to known medicinal species, with little consideration of phylogenetic relatedness except in few recent research concerning Euphorbiaceae (Ernst et al., 2018). However, with the existence of phylogenetic and metabolomic information across multiple species, this approach could be further scaled up to comprising taxa with ethnobotanic medicinal use as well as their sister taxa for which no known records of ethnobotanical medicinal use exist, to identify target taxa for medicinal potential (see Figure 3).

In summary, the combination of ethnobotanic information, phylogeny and molecular networking provides a promising approach to plant natural product-based drug discovery. By investigating the metabolomics profiles, of a target highly bioactive plant clade comprising both known medicinal and understudied taxa, chemically related taxa could be identified via molecular networking. The phylogenetic signal of those chemical compounds (or chemical networks) could be quantitatively measured by constructing the phylogeny and using phylogenetic signal-measuring statistical methods. This will allow prediction of the medicinal potential of previously understudied taxa. Combining these approaches holds a key to the advancement of plant derived natural product drug discovery.

\section{AUTHOR CONTRIBUTIONS}

SM did the literature survey and wrote the paper. MS designed the research and edited the paper. DC edited and provided metabolomic insight to the paper. AG and NR edited the paper. networking and docking strategies for the dereplication of active compounds. J. Pharm. Biomed. Anal. 158, 471-479. doi: 10.1016/j.jpba.2018.06.026

Acamovic, T., and Brooker, J. D. (2005). Biochemistry of plant secondary metabolites and their effects in animals. Proc. Nutr. Soc. 64, 403-412. doi: 10.1079/PNS2005449 
Afendi, F. M., Okada, T., Yamazaki, M., Hirai-Morita, A., Nakamura, Y., Nakamura, K., et al. (2012). Knapsack family databases: integrated metaboliteplant species databases for multifaceted plant research. Plant Cell Physiol. 53, e1-e1. doi: 10.1093/pcp/pcr165

Agrawal, A. A., Hastings, A. P., Johnson, M. T. J., Maron, J. L., and Salminen, J. P. (2012a). Insect herbivores drive real-time ecological and evolutionary change in plant populations. Science 338, 113-116. doi: 10.1126/science.1225977

Agrawal, A. A., Petschenka, G., Bingham, R. A., Weber, G. M., and Rasmann, S. (2012b). Toxic cardenolides: chemical ecology and coevolution of specialized plant-herbivore interactions. New Phytol. 194, 28. doi: 10.1111/j.1469-8137.2011.04049.x

Allard, P. M., Péresse, T., Bisson, J., Gindro, K., Marcourt, L., Pham, V. C., et al. (2016). Integration of molecular networking and in-silico $\mathrm{ms} / \mathrm{ms}$ fragmentation for natural products dereplication. Anal. Chem. 88, 3317-3323. doi: 10.1021/acs.analchem.5b04804

Allkin, B. (2017). "Useful medicines," in State of the World's Plants 2017, eds K. J. Willis (London: The Board of Trustees of the Royal Botanic Gardens, Royal Botanic Gardens, Kew), 22-29.

Atanasov, A. G., Waltenberger, B., Pferschy-Wenzig, E. M., Linder, T., Wawrosch, C., Uhrin, P., et al. (2015). Discovery and resupply of pharmacologically active plant-derived natural products: a review. Biotechnol. Adv. 33, 1582-1614. doi: 10.1016/j.biotechadv.2015.08.001

Auffret, M., Drapier, S., and Vérin, M. (2018). The many faces of apomorphine: Lessons from the past and challenges for the future. Drugs $R$ D 18, 91-107. doi: 10.1007/s40268-018-0230-3

Baloglu, E., and Kingston, D. G. I. (1999). A new semisynthesis of paclitaxel from baccatin iii. J. Nat. Products 62, 1068-1071.

Balunas, M. J., and Kinghorn, A. D. (2005). Drug discovery from medicinal plants. Life Sci. 78, 431-441. doi: 10.1016/j.lfs.2005.09.012

Benderoth, M., Textor, S., Windsor, A. J., Mitchell-Olds, T., Gershenzon, J., and Kroymann, J. (2006). Positive selection driving diversification in plant secondary metabolism. Proc. Nat. Acad. Sci. 103, 9118-9123. doi: $10.1073 /$ pnas.0601738103

Berenbaum, M. R. (2001). Chemical mediation of coevolution: Phylogenetic evidence for apiaceae and associates. Ann. Missouri Botan. Garden 88, 45-59. doi: $10.2307 / 2666131$

Berenbaum, M. R., and Zangerl, A. R. (2008). Facing the future of plant-insect interaction research: Le retour à la "raison d'être". Plant Physiol. 146, 804-811. doi: $10.1104 /$ pp.107.113472

Blomberg, S. P., Garland, T Jr., and Ives, A. R. (2003). Testing for phylogenetic signal in comparative data: Behavioral traits are more labile. Evolution 57, 717-745. doi: 10.1111/j.0014-3820.2003.tb00285.x

Bonaventure, G., Vandoorn, A., and Baldwin, I. T. (2011). Herbivore-associated elicitors: Fac signaling and metabolism. Trends Plant Sci. 16, 294-299. doi: 10.1016/j.tplants.2011.01.006

Caseys, C., Stritt, C., Glauser, G., Blanchard, T., and Lexer, C. (2015). Effects of hybridization and evolutionary constraints on secondary metabolites: the genetic architecture of phenylpropanoids in European Populus species. PLoS ONE 10:e0128200. doi: 10.1371/journal.pone. 0128200

Chae, L., Kim, T., Nilo-Poyanco, R., and Rhee, S. Y. (2014). Genomic signatures of specialized metabolism in plants. Science 344, 510-513. doi: $10.1126 /$ science. 1252076

Chezem, W. R., and Clay, N. K. (2016). Regulation of plant secondary metabolism and associated specialized cell development by mybs and bhlhs. Phytochemistry 131, 26-43. doi: 10.1016/j.phytochem.2016.08.006

Coley, P. D., Heller, M. V., Aizprua, R., Araúz, B., Flores, N., Correa, M., et al. (2003). Using ecological criteria to design plant collection strategies for drug discovery. Front. Ecol. Environ. 1, 421-428. doi: 10.1890/1540-9295(2003)001[0421:UECTDP].0.CO

Courtois, E. A., Dexter, K. G., Paine, C. E., Stien, D., Engel, J., Baraloto, C., et al. (2016). Evolutionary patterns of volatile terpene emissions across 202 tropical tree species. Ecol. Evol. 6, 2854-2864. doi: 10.1002/ec e3.1810

Das, A. M. (2017). Clinical utility of nitisinone for the treatment of hereditary tyrosinemia type-1 (ht-1). Appl. Clin. Genet. 10, 43-48. doi: 10.2147/TACG.S113310
De Luca, V., Salim, V., Atsumi, S. M., and Yu, F. (2012). Mining the biodiversity of plants: a revolution in the making. Science 336, 1658-1661. doi: $10.1126 /$ science. 1217410

De Medeiros, P. M., Ladio, A. H., and Albuquerque, U. P. (2015). "Local criteria for medicinal plant selection," in Evolutionary Ethnobiology, eds U. P. Albuquerque, P. M. De Medeiros, A. Casas (Cham: Springer International Publishing). doi: 10.1007/978-3-319-19917-7_11

Dias, A. D., Jones, A. O., Beale, J. D., Boughton, A. B., Benheim, D., Kouremenos, A. K., et al. (2016). Current and future perspectives on the structural identification of small molecules in biological systems. Metabolites 6:E46. doi: 10.3390/metabo6040046

Dias, D. A., Urban, S., and Roessner, U. (2012). A historical overview of natural products in drug discovery. Metabolites 2, 303-336. doi: 10.3390/metabo2020303

Edger, P. P., Heidel-Fischer, H. M., Bekaert, M., Rota, J., Glöckner, G., Platts, A. E., et al. (2015). The butterfly plant arms-race escalated by gene and genome duplications. Proc. Nat. Acad. Sci. 112, 8362-8366. doi: $10.1073 /$ pnas. 1503926112

Ehrlich, P. R., and Raven, P. H. (1964). Butterflies and plants: a study in coevolution. Evolution 18, 586-608. doi: 10.1111/j.1558-5646.1964.tb01674.x

Ernst, M., Nothias-Scaglia, L. F., Van Der Hooft, J., Silva, R. R., Saslis-Lagoudakis, C. H., Grace, O. M., et al. (2018). Did a plant-herbivore arms race drive chemical diversity in euphorbia? BioRxiv 323014. doi: 10.1101/323014

Ernst, M., Saslis-Lagoudakis, C. H., Grace, O. M., Nilsson, N., Simonsen, H. T., Horn, J. W., et al. (2016). Evolutionary prediction of medicinal properties in the genus euphorbia 1. Sci. Rep. 6:30531. doi: 10.1038/srep30531

Feng, C., Dorothea, T., Jörg, B., and Eran, P. (2011). The family of terpene synthases in plants: A mid-size family of genes for specialized metabolism that is highly diversified throughout the kingdom. Plant J. 66, 212-229. doi: 10.1111/j.1365-313X.2011.04520.x

Figueiredo, A. C., Barroso, J. G., Pedro, L. G., and Scheffer, J. J. C. (2008). Factors affecting secondary metabolite production in plants: Volatile components and essential oils. Flavour Frag J. 23, 213-226. doi: 10.1002/ffj.1875

Fritz, S. A., and Purvis, A. (2010). Selectivity in mammalian extinction risk and threat types: a new measure of phylogenetic signal strength in binary traits. Conserv. Biol. 24, 1042-1051. doi: 10.1111/j.1523-1739.2010.01455.x

Garnatje, T., Peñuelas, J., and Vallès, J. (2017). Ethnobotany, phylogeny, and 'omics' for human health and food security. Trends Plant Sci. 22, 187-191. doi: 10.1016/j.tplants.2017.01.001

Gouinguen,é, S. P., and Turlings, T. C. J. (2002). The effects of abiotic factors on induced volatile emissions in corn plants. Plant Physiol. 129, 1296-1307. doi: $10.1104 /$ pp.001941

Grace, O. M., Buerki, S., Symonds, M. R. E., Forest, F., Van Wyk, A. E., Smith, G. F., et al. (2015). Evolutionary history and leaf succulence as explanations for medicinal use in aloes and the global popularity of aloe vera. BMC Evolution. Biol. 15:29. doi: 10.1186/s12862-015-0291-7

Gupta, R., Gabrielsen, B., and Ferguson, S. M. (2005). Nature's medicines: Traditional knowledge and intellectual property management. Case studies from the national institutes of health (nih), USA. Curr. Drug Discov. Technol. 2, 203-219. doi: 10.2174/157016305775202937

Gurib-Fakim, A. (2006). Medicinal plants: traditions of yesterday and drugs of tomorrow. Mol Aspects Med. 27, 1-93. doi: 10.1016/j.mam.2005.07.008

Guzman, E., and Molina, J. (2018). The predictive utility of the plant phylogeny in identifying sources of cardiovascular drugs. Pharmaceut. Biol. 56, 154-164. doi: 10.1080/13880209.2018.1444642

Halberstein, R. A. (2005). Medicinal plants: historical and cross-cultural usage patterns. Ann. Epidemiol. 15, 686-699. doi: 10.1016/j.annepidem.2005. 02.004

Hartmann, T. (2007). From waste products to ecochemicals: fifty years research of plant secondary metabolism. Phytochemistry 68, 2831-2846. doi: 10.1016/j.phytochem.2007.09.017

Harvey, A. L., Edrada-Ebel, R., and Quinn, R. J. (2015). The re-emergence of natural products for drug discovery in the genomics era. Nat. Rev. Drug Discov. 14, 111-129. doi: 10.1038/nrd4510

Heinrich, M., Edwards, S., Moerman, D. E., and Leonti, M. (2009) Ethnopharmacological field studies: a critical assessment of their conceptual basis and methods. J. Ethnopharmacol. 124, 1-17. doi: 10.1016/j.jep.2009.03.043 
Holton, R. A., Somoza, C., Kim, H. B., Liang, F., Biediger, R. J., Boatman, P. D., et al. (1994). First total synthesis of taxol. 1. Functionalization of the B ring. J. Am. Chem. Soc. 116, 1597-1598.

Johnson, S. R., and Lange, B. M. (2015). Open-access metabolomics databases for natural product research: present capabilities and future potential. Front. Bioeng. Biotechnol. 3, 22-22. doi: 10.3389/fbioe.2015.00022

Kallenbach, M., Alagna, F., Baldwin, I. T., and Bonaventure, G. (2010). nicotiana attenuata sipk, wipk, nprl, and fatty acid-amino acid conjugates participate in the induction of jasmonic acid biosynthesis by affecting early enzymatic steps in the pathway. Plant Physiol. 152, 96-106. doi: 10.1104/pp.109.149013

Kamilar, J. M., and Cooper, N. (2013). Phylogenetic signal in primate behaviour, ecology and life history. Philos. Trans. R Soc. B Biol. Sci. 368:20120341. doi: $10.1098 /$ rstb.2012.0341

Kingston, D. G. I. (2011). Modern natural products drug discovery and its relevance to biodiversity conservation. J. Nat. Products 74, 496-511. doi: $10.1021 / \mathrm{np} 100550 \mathrm{t}$

Kleigrewe, K., Almaliti, J., Tian, I. Y., Kinnel, R. B., Korobeynikov, A., Monroe, E. A., et al. (2015). Combining mass spectrometric metabolic profiling with genomic analysis: a powerful approach for discovering natural products from cyanobacteria. J. Nat. Prod. 78, 1671-1682. doi: 10.1021/acs.jnatprod.5b00301

Kowiyou, Y. H., Daru, B. H., and Muthama, M. A. (2015). Phylogenetic exploration of commonly used medicinal plants in south africa. Mol. Ecol. Res. 15, 405-413. doi: 10.1111/1755-0998.12310

Leonti, M., and Casu, L. (2013). Traditional medicines and globalization: current and future perspectives in ethnopharmacology. Front. Pharmacol. 4, 92-92. doi: 10.3389/fphar.2013.00092

Li, J. W. H., and Vederas, J. C. (2009). Drug discovery and natural products: end of an era or an endless frontier? Science 325, 161-165. doi: 10.1126/science.1168243

Liu, Y., and Wang, M. W. (2008). Botanical drugs: challenges and opportunities: contribution to linnaeus memorial symposium 2007. Life Sci. 82, 445-449. doi: 10.1016/j.lfs.2007.11.007

Mcgonigle, I. V. (2016). Patenting nature or protecting culture? ethnopharmacology and indigenous intellectual property rights. J. Law Biosci. 3, 217-226. doi: 10.1093/jlb/lsw003

Meldau, S., Baldwin, I. T., and Wu, J. (2011). Sgtl regulates wounding- and herbivory-induced jasmonic acid accumulation and nicotiana attenuata's resistance to the specialist lepidopteran herbivore manduca sexta. New Phytologist. 189, 1143-1156. doi: 10.1111/j.1469-8137.2010.03558.x

Müller, C., and Orians, C. M. (2018). From plants to herbivores: novel insights into the ecological and evolutionary consequences of plant variation. Oecologia 187, 357-360. doi: 10.1007/s00442-018-4126-2

Münkemüller, T., Lavergne, S., Bzeznik, B., Dray, S., Jombart, T., Schiffers, K., et al. (2012). How to measure and test phylogenetic signal. Methods Ecol. Evol. 3, 743-756. doi: 10.1111/j.2041-210X.2012.00196.x

Newman, D. J., and Cragg, G. M. (2016). Natural products as sources of new drugs from 1981 to 2014. J. Nat. Products 79, 629-661. doi: 10.1021/acs.jnatprod.5b01055

Nothias, L. F., Nothias-Esposito, M., Da Silva, R., Wang, M., Protsyuk, I., Zhang, Z., et al. (2018). Bioactivity-based molecular networking for the discovery of drug leads in natural product bioassay-guided fractionation. J. Nat. Products 81, 758-767. doi: 10.1021/acs.jnatprod.7b00737

Olivon, F., Apel, C., Retailleau, P., Allard, P. M., Wolfender, J. L., Touboul, D., et al. (2018). Searching for original natural products by molecular networking: Detection, isolation and total synthesis of chloroaustralasines. Org. Chem. Front. 5, 2171-2178. doi: 10.1039/C8QO00429C

Pellicer, J., Saslis-Lagoudakis, C. H., Carrió, E., Ernst, M., Garnatje, T., Grace, O. M., et al. (2018). A phylogenetic road map to antimalarial artemisia species. J. Ethnopharmacol. 225, 1-9. doi: 10.1016/j.jep.2018.06.030

Pert, P. L., Ens, E. J., Locke, J., Clarke, P. A., Packer, J. M., and Turpin, G. (2015). An online spatial database of australian indigenous biocultural knowledge for contemporary natural and cultural resource management. Sci. Total Environ. 534, 110-121. doi: 10.1016/j.scitotenv.2015.01.073

Rates, S. M. (2001). Plants as source of drugs. Toxicon 39, 603-613. doi: 10.1016/S0041-0101(00)00154-9

Reigosa, M. J., and Pazos-Malvido, E. (2007). Phytotoxic effects of 21 plant secondary metabolites on arabidopsis thaliana germination and root growth. J. Chem. Ecol. 33, 1456-1466. doi: 10.1007/s10886-007-9318-x
Roersch, C. M. F. B. (2010). Piper umbellatum l.: A comparative cross-cultural analysis of its medicinal uses and an ethnopharmacological evaluation. $J$. Ethnopharmacol. 131, 522-537. doi: 10.1016/j.jep.2010.07.045

Rønsted, N., Savolainen, V., Mølgaard, P., and Jäger, A. K. (2008). Phylogenetic selection of narcissus species for drug discovery. Biochem. Systemat. Ecol. 36, 417-422. doi: 10.1016/j.bse.2007.12.010

Rønsted, N., Symonds, M. R. E., Birkholm, T., Christensen, S. B., Meerow, A. W., Molander, M., et al. (2012). Can phylogeny predict chemical diversity andpotential medicinal activity of plants? a case study of amaryllidaceae. BMC Evolut. Biol. 12:182. doi: 10.1186/1471-2148-12-182

Salazar, D., Lokvam, J., Mesones, I., Vásquez Pilco, M., Ayarza Zuñiga, J. M., De Valpine, P., et al. (2018). Origin and maintenance of chemical diversity in a species-rich tropical tree lineage. Nat. Ecol. Evol. 2, 983-990. doi: 10.1038/s41559-018-0552-0

Saslis-Lagoudakis, C. H., Klitgaard, B. B., Forest, F., Francis, L., Savolainen, V., Williamson, E. M., et al. (2011). The use of phylogeny to interpret cross-cultural patterns in plant use and guide medicinal plant discovery: An example from pterocarpus (leguminosae). PLoS ONE. 6:e22275. doi: 10.1371/journal.pone.0022275

Saslis-Lagoudakis, C. H., Rønsted, N., Clarke, A. C., and Hawkins, J. A. (2015). "Evolutionary approaches to ethnobiology," in Evolutionary Ethnobiology, eds U. P. Albuquerque, P. M. De Medeiros, A Casas. (Cham: Springer International Publishing). doi: 10.1007/978-3-319-19917-7_5

Saslis-Lagoudakis, C. H., Savolainen, V., Williamson, E. M., Forest, F., Wagstaff, S. J., Baral, S. R., et al. (2012). Phylogenies reveal predictive power of traditional medicine in bioprospecting. Proc. Nat. Acad. Sci. 109, 15835-15840. doi: 10.1073/pnas.1202242109

Schwager, J., Mohajeri, M. H., Fowler, A., and Weber, P. (2008). Challenges in discovering bioactives for the food industry. Curr. Opin. Biotechnol. 19, 66-72. doi: 10.1016/j.copbio.2008.02.016

Shane, G. (2004). Indigenous people incorporated? : culture as politics, culture as property in pharmaceutical bioprospecting1. Curr. Anthropol. 45, 211-237. doi: $10.1086 / 381047$

Speed, M. P., Fenton, A., Jones, M. G., Ruxton, G. D., and Brockhurst, M. A. (2015). Coevolution can explain defensive secondary metabolite diversity in plants. New Phytologist. 208, 1251-1263. doi: 10.1111/nph.13560

Takeuchi, M., Saito, Y., Goto, M., Miyake, K., Newman, D. J., O'keefe, B. R., et al. (2018). Antiproliferative alkaloids from alangium longiflorum, an endangered tropical plant species. J. Nat. Products 81, 1884-1891. doi: 10.1021/acs.jnatprod.8b00411

Toju, H., and Sota, T. (2006). Imbalance of predator and prey armament: geographic clines in phenotypic interface and natural selection. Am. Nat. 167, 105-117. doi: 10.1086/498277

$\mathrm{Tu}, \mathrm{Y}$. (2011). The discovery of artemisinin (qinghaosu) and gifts from chinese medicine. NatMed. 17, 1217-1220. doi: 10.1038/nm.2471

Wagner, H. (2011). Synergy research: approaching a new generation of phytopharmaceuticals. Fitoterapia 82, 34-37. doi: 10.1016/j.fitote.2010.11.016

Wall, M. E., and Wani, M. C. (1995). Camptothecin and taxol: discovery to clinicthirteenth Bruce F. Cain Memorial Award Lecture. Cancer Res. 55, 753-760.

Wani, M. C., Taylor, H. L., Wall, M. E., Coggon, P., and Mcphail, A. T. (1971). Plant antitumor agents. VI. Isolation and structure of taxol, a novel antileukemic and antitumor agent from Taxus brevifolia. J. Am. Chem. Soc. 93, 2325-2327.

Waterman, J. M., Cazzonelli, C. I., Hartley, S. E., and Johnson, S. N. (2019). Simulated herbivory: the key to disentangling plant defence responses. Trends Ecol. Evolut. 34, 447-458. doi: 10.1016/j.tree.2019.01.008

Weaver, B. A. (2014). How taxol/paclitaxel kills cancer cells. Mol. Biol. Cell 25, 2677-2681. doi: 10.1091/mbc.E14-04-0916

Wells, T. N. C., Van Huijsduijnen, R. H., and Van Voorhis, W. C. (2015). Malaria medicines: a glass half full? Nat. Rev. Drug Discov. 424. doi: 10.1038/ $\operatorname{nrd} 4573$

Willis, K. J. (2017). “State of the world's plants 2017," in State of the World's Plants, eds W. Kj. (London: Royal Botanic Gardens, Kew).

Wink, M. (1988). Plant breeding: importance of plant secondary metabolites for protection against pathogens and herbivores. Theoret. Appl. Genet. 75, 225-233. doi: $10.1007 / \mathrm{BF} 00303957$

Wink, M. (2003). Evolution of secondary metabolites from an ecological and molecular phylogenetic perspective. Phytochemistry 64, 3-19. doi: 10.1016/S0031-9422(03)00300-5 
Wink, M. (2015). Modes of action of herbal medicines and plant secondary metabolites. Medicines 2, 251-286. doi: 10.3390/medicines20 30251

Wu, J., Hettenhausen, C., Meldau, S., and Baldwin, I. T. (2007). Herbivory rapidly activates mapk signaling in attacked and unattacked leaf regions but not between leaves of nicotiana attenuata. Plant Cell 19, 1096-1122. doi: 10.1105/tpc.106. 049353

Zangerl, A. R., Berenbaum, M. R., and Mallet, J. (2003). Phenotype matching in wild parsnip and parsnip webworms: causes and consequences. Evol. 57, 806-815. doi: 10.1111/j.0014-3820.2003.tb 00292.x

Zhu, F., Qin, C., Tao, L., Liu, X., Shi, Z., Ma, X., et al. (2011). Clustered patterns of species origins of nature-derived drugs and clues for future bioprospecting.
Proc. Nat. Acad. Sci USA. 108, 12943-12948. doi: 10.1073/pnas.11073 36108

Conflict of Interest Statement: The authors declare that the research was conducted in the absence of any commercial or financial relationships that could be construed as a potential conflict of interest.

Copyright $\odot 2019$ Mawalagedera, Callahan, Gaskett, Rønsted and Symonds. This is an open-access article distributed under the terms of the Creative Commons Attribution License (CC BY). The use, distribution or reproduction in other forums is permitted, provided the original author $(s)$ and the copyright owner(s) are credited and that the original publication in this journal is cited, in accordance with accepted academic practice. No use, distribution or reproduction is permitted which does not comply with these terms. 